\title{
Circulating sortilin level as a potential biomarker for coronary atherosclerosis and diabetes mellitus
}

\author{
Tae Jung Oh ${ }^{1,2}$, Chang Ho Ahn', Bo-Rahm Kim,2, Kyoung Min Kim 1,2, Jae Hoon Moon 1,2, Soo Lim,2, \\ Kyong Soo Park', Cheong Lim ${ }^{3,4}$, HakChul Jang ${ }^{1,2}$ and Sung Hee Choi ${ }^{1,2^{*}}$ (i)
}

\begin{abstract}
Context: A previous genome-wide association study showed that a genetic variant of sortilin was associated with the risk of coronary artery disease (CAD). However, the role of circulating sortilin is still unknown. We investigated the potential role of plasma sortilin as a biomarker for CAD and diabetes mellitus.

Methods: We enrolled statin-naive subjects with CAD $(n=31)$ who underwent coronary artery bypass surgery and control subjects $(n=116)$ who were free from CAD as evaluated by coronary CT angiography. The presence of diabetes mellitus was evaluated and plasma sortilin levels were measured with a commercial ELISA kit.

Results: Plasma sortilin levels were higher in subjects with CAD and subjects with diabetes mellitus than in those without CAD or diabetes mellitus. Subjects in the highest sortilin tertile group were older and had higher glucose and $\mathrm{HbA1c}$ levels, but lipid profiles in the three tertile groups were comparable. Multivariable logistic regression analysis revealed that sortilin levels were independently associated with CAD. In addition, the receiver operating characteristic curve analysis showed that plasma sortilin levels could identify the presence of CAD or diabetes mellitus.
\end{abstract}

Conclusions: Elevated circulating sortilin levels are associated with CAD and diabetes mellitus and can be used as a biomarker of both diseases in statin-naïve subjects.

Keywords: Coronary artery disease, Diabetes mellitus, Sortilin, Proneurotensin, Biomarker

\section{Background}

The number of adults with diabetes mellitus has nearly quadrupled in the USA over the past 30 years [1]. In addition, it is estimated that the global prevalence of diabetes mellitus will increase by $54 \%$ from 2010 to 2030 [2]. In Korea, the prevalence of diabetes mellitus is also increasing and the last national survey showed that the prevalence among adults 30 years or older is $13.7 \%$ [3]. However, the majority of individuals with diabetes are not aware of their condition and the rate may be up to $30 \%$ [3]. Therefore, biomarkers for diabetes mellitus

\footnotetext{
*Correspondence: drshchoi@snu.ac.kr

1 Department of Internal Medicine, Seoul National University College of Medicine, Seoul, South Korea

Full list of author information is available at the end of the article
}

should be developed to improve the identification of high-risk subjects.

In patients with diabetes mellitus, the leading cause of death is cardiovascular disease, even though the rates of cardiovascular disease declined between 1990 and 2010 [4]. It is unquestionable that diabetes mellitus is the most important risk factor for cardiovascular disease [5]. In this context, a biomarker for diabetes mellitus could be used for the prediction of CAD and vice versa. In fact, there are common shared biomarkers for CAD and diabetes mellitus [6, 7]. These biomarkers are useful for distinguishing patients at high risk for $\mathrm{CAD}[3,8]$ and may be good candidates as therapeutic targets. In this context, investigation of biomarkers for both CAD and diabetes mellitus has important clinical implications.

The Wellcome Trust Case Control Consortium study showed that a single nucleotide polymorphism at the 
$1 \mathrm{p} 13$ locus was associated with the risk of CAD [9]. Subsequent studies demonstrated that the SORT1 gene was associated with hepatic lipid metabolism [10] and lowdensity lipoprotein (LDL)-cholesterol levels [11]. The SORT1 gene encodes sortilin, which belongs to the mammalian vacuolar protein sorting 10 protein domain family [12]. Sortilin is located mainly within cells in sites such as the trans-Golgi network, and it is also located in the cell membrane [12] and systemic circulation [13]. Few studies have reported that soluble sortilin and the propeptide fragment of its ligand, proneurotensin, were associated with cardiovascular risk factors $[14,15]$. Ogawa demonstrated that soluble sortilin levels were positively associated with cardiovascular risk factors, but that soluble sortilin levels were lower in patients with CAD than controls [14]. In another study from the Framingham Heart Study, increased circulating proneurotensin was associated with a higher rate of cardiovascular events [15]. Therefore, there is some discrepancy between the two studies of prediction of CVD using these two circulating molecules related to sortilin. In this study, we measured circulating sortilin levels, and tested whether the measurement of circulating sortilin can be used as a biomarker of both CVD and diabetes mellitus.

\section{Methods}

\section{Study population}

We enrolled subjects with and without confirmed CAD. The subjects with CAD were recruited from the Thoracic Surgery Department of the Seoul National University Bundang Hospital (SNUBH). They underwent coronary artery bypass graft (CABG) surgery because of acute myocardial infarction, unstable angina, or stable angina with three-vessel disease. The subjects that were free from $\mathrm{CAD}$, as evaluated by coronary $\mathrm{CT}$ angiography, were recruited from the outpatient clinic of Endocrinology Department at the same hospital. We excluded subjects who had been treated with statins within the previous 3 months or whose medical records were not available. A total of 31 subjects with CAD and 116 subjects without CAD were enrolled from May 2012 to January 2015. Of these, 43 (29.3\%) subjects were diagnosed with diabetes mellitus. The study was approved by the Institutional Review Board of the SNUBH (IRB No B1203/147-006) and all subjects gave informed consent.

\section{Medical history and diagnosis}

We defined $\mathrm{CAD}$ as acute myocardial infarction or angina pectoris that needed to be treated with CABG surgery. The definition of normal coronary arteries was the absence of luminal stenosis of $\geq 50 \%$ with a coronary artery calcium score $<10$. Diabetes mellitus was defined as a fasting plasma glucose $\geq 126 \mathrm{mg} / \mathrm{dl}$ and/or $\mathrm{HbA} 1 \mathrm{c}$ $\geq 6.5 \%$ or treatment with antidiabetes medication. Medical records were reviewed and medical history and clinical characteristics such as age, sex, body weight, height, systolic and diastolic blood pressure were obtained. Biochemical analysis was performed immediately before surgery or within 3 months of coronary CT angiography. Nonfasting blood samples were obtained and centrifuged at $3000 \mathrm{rpm}$ for $10 \mathrm{~min}$ at $4{ }^{\circ} \mathrm{C}$. The plasma was stored at $-80^{\circ} \mathrm{C}$ until analysis.

\section{Biochemical assays}

The plasma levels of glucose and HbA1c were measured by the hexokinase method and high-performance liquid chromatography, respectively. Total cholesterol, triglyceride, high-density lipoprotein (HDL)-cholesterol, and LDL-cholesterol levels were measured by relevant enzymatic assays and the glycerol-3-phosphate oxidase peroxide method. The high-sensitive $\mathrm{C}$-reactive protein (hs-CRP) was measured by latex-enhanced turbidometric immunoassay (Denka Seiken Co., Tokyo, Japan). These laboratory analyses were performed by the central laboratory of SNUBH. Circulating plasma sortilin was measured using a commercial enzyme-linked immunosorbent assay (ELISA) (Cusabio ELISA kits, Cosmo Bio, Carlsbad, CA, USA). This ELISA kit can detect sortilin levels of $46.88-3000 \mathrm{pg} / \mathrm{ml}$ with a coefficient of variation $<8 \%$ for intra-assay precision and $<10 \%$ for interassay variation.

\section{Statistical analysis}

Continuous variables are presented as mean and standard deviation and categorical variables are presented as percentages. The differences between two groups were analyzed by 2-tailed Student $t$ test or nonparametric $t$ test and age-adjusted analysis of covariance. Categorical variables were compared using the Chi square test. Comparison between the three tertile groups was performed using one-way analysis of variance (ANOVA) followed by least significant difference (LSD) post hoc analysis. A log transformation was applied to glucose, HbA1c, triglyceride, hs-CRP, and sortilin levels prior to performing ANOVA and logistic regression analysis. The association with CAD or diabetes mellitus was evaluated by univariable and multivariable logistic regression analysis. The area under the receiver-operating characteristics (ROC) curve was calculated to test its predictive discrimination of CAD. The optimal cut-off value was determined using the maximum sum of sensitivity and specificity based on the Youden-Index. Statistical analysis was performed using IBM SPSS Statistics for Windows, version 20.0 (IBM Corp., Armonk, NY, USA). For all tests, $P<0.05$ was considered to be significant. 


\section{Results}

The clinical and biochemical characteristics of subjects with or without CAD are shown in Table 1 . Subjects with CAD were older and had higher systolic blood pressure and blood glucose levels than their counterparts. They showed lower HDL- and LDL-cholesterol levels than subjects without CAD and included a higher proportion of subjects diagnosed with diabetes mellitus, hypertension, and dyslipidemia. Circulating plasma sortilin levels were higher in subjects with CAD than those without CAD (Fig. 1). The significance remained after age adjustment. Two subjects that had extreme values for sortilin $(6.47$ and $6.75 \mathrm{ng} / \mathrm{ml})$ did not have CAD. However, when we eliminated these two outliers the study results were not changed. Overall, 26 of 31 subjects with CAD took aspirin. We did not see any significant difference in circulating sortilin levels between aspirin users and non-users $(1.72 \pm 0.45$ vs. $1.86 \pm 0.42 \mathrm{ng} / \mathrm{ml}, P=0.548)$.

Next, we compared the variables in subjects with or without diabetes mellitus (Table 2). The subjects with diabetes mellitus showed similar clinical deterioration to the subjects with CAD. The sortilin levels were significantly higher in subjects with diabetes mellitus compared with those without diabetes mellitus $(1.57 \pm 0.54$, and $1.34 \pm 1.0 \mathrm{ng} / \mathrm{ml}, P=0.006$ ).

Table 3 shows the general characteristics of study participants grouped according to sortilin tertiles. The mean age of subjects in tertile 1 was lower than those in tertiles

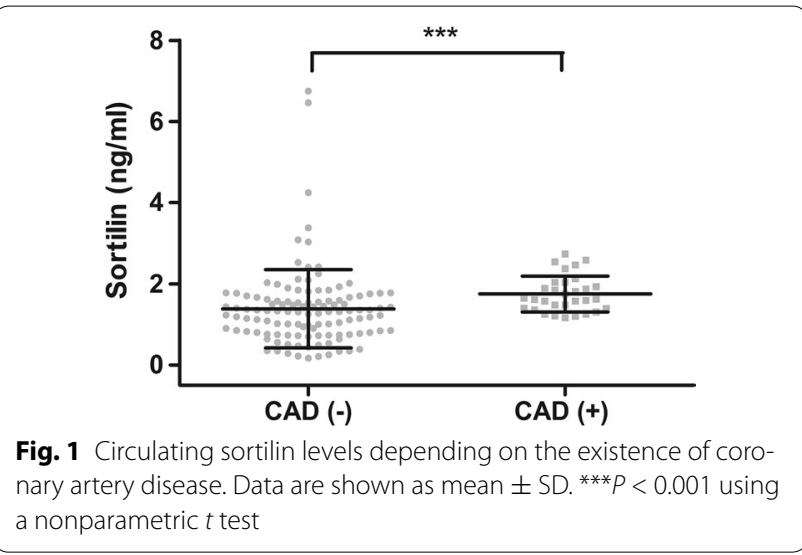

2 and 3. Body weight, blood pressure, and lipid profiles were comparable between the three tertile groups. However, blood glucose and HbA1c levels were higher in subjects in tertiles 2 and 3 than in subjects in tertile 1 . In addition, more subjects in tertile 3 were diagnosed as having diabetes mellitus and CAD.

As shown in Table 4, univariable logistic regression analysis revealed that age, systolic blood pressure, log glucose, log HbA1c, total cholesterol, HDL-cholesterol, LDL-cholesterol, log hs-CRP, and log sortilin were associated with CAD. We included these variables in multivariable logistic regression analysis but omitted log glucose,

Table 1 Clinical and biochemical characteristics of study participants classified according to coronary artery disease

\begin{tabular}{|c|c|c|c|c|c|}
\hline & Total subjects & CAD (-), $n=116$ & CAD $(+), n=31$ & $P$ value & $P$ value* \\
\hline Age (years) & $55.5 \pm 11.0$ & $52.9 \pm 9.2$ & $65.4 \pm 11.8$ & $<0.001$ & $\mathrm{~N} / \mathrm{a}$ \\
\hline Men (\%) & 69.4 & 66 & 84 & 0.052 & $\mathrm{~N} / \mathrm{a}$ \\
\hline Body weight (kg) & $65.3 \pm 11.0$ & $65.8 \pm 11.5$ & $63.3 \pm 8.60$ & 0.203 & 0.886 \\
\hline $\operatorname{BMI}\left(\mathrm{kg} / \mathrm{m}^{2}\right)$ & $23.9 \pm 2.8$ & $24.1 \pm 2.9$ & $23.4 \pm 2.5$ & 0.213 & 0.587 \\
\hline $\mathrm{SBP}(\mathrm{mmHg})$ & $122.2 \pm 17.0$ & $120.5 \pm 16.4$ & $128.6 \pm 18.0$ & 0.019 & 0.015 \\
\hline $\mathrm{DBP}(\mathrm{mmHg})$ & $73.7 \pm 11.7$ & $74.1 \pm 12.2$ & $72.3 \pm 9.5$ & 0.438 & 0.633 \\
\hline Glucose (mg/dl) & $107.0 \pm 1.4$ & $102.0 \pm 30.9$ & $161.0 \pm 77.0$ & $<0.001$ & $<0.001$ \\
\hline $\mathrm{HbA1c}(\%)$ & $6.2 \pm 1.2$ & $6.2 \pm 1.4$ & $6.9 \pm 1.5$ & 0.004 & 0.068 \\
\hline $\mathrm{TC}(\mathrm{mg} / \mathrm{dl})$ & $194.0 \pm 40.3$ & $202.1 \pm 35.3$ & $164.0 \pm 43.9$ & $<0.001$ & $<0.001$ \\
\hline LDL-C (mg/dl) & $110.1 \pm 26.8$ & $112.6 \pm 26.0$ & $100.6 \pm 28.4$ & 0.026 & 0.097 \\
\hline HDL-C (mg/dl) & $49.1 \pm 12.3$ & $52.0 \pm 11.7$ & $38.1 \pm 7.2$ & $<0.001$ & $<0.001$ \\
\hline TG (mg/dl) & $132.7 \pm 66.9$ & $131.9 \pm 67.4$ & $135.6 \pm 65.9$ & 0.623 & 0.345 \\
\hline hs-CRP (mg/dl) & $0.28 \pm 0.71$ & $0.11 \pm 0.22$ & $0.87 \pm 1.26$ & $<0.001$ & $<0.001$ \\
\hline $\mathrm{DM}(\%)$ & 29.3 & 19.8 & 64.5 & $<0.001$ & N/a \\
\hline HTN (\%) & 53.7 & 43.1 & 93.5 & $<0.001$ & $\mathrm{~N} / \mathrm{a}$ \\
\hline Dyslipidemia (\%) & 37.4 & 28.4 & 71.0 & $<0.001$ & N/a \\
\hline
\end{tabular}

Data are expressed as mean $\pm \mathrm{SD}$, or \%. CAD coronary artery disease, $D M$ diabetes mellitus, $B M I$ body mass index, $S B P$ systolic blood pressure, $D B P$ diastolic blood pressure, TC total cholesterol, LDL-C LDL cholesterol, HDL-C HDL cholesterol, TG triglyceride, HTN hypertension

$P$ values for student's $t$ test or nonparametric $t$ test or Chi square test

* $P$ values for age-adjusted ANCOVA 
Table 2 Clinical and biochemical characteristics and sortilin levels in subgroup without coronary artery disease according to their diabetes mellitus status

\begin{tabular}{lllr}
\hline & DM $(-\mathbf{l}, \mathbf{n}=\mathbf{9 3}$ & DM $(+), \mathbf{n}=\mathbf{2 3}$ & $\boldsymbol{P}$ value \\
\hline Age (years) & $52.5 \pm 8.3$ & $54.5 \pm 12.0$ & 0.444 \\
Men (\%) & 31.2 & 47.8 & 0.148 \\
Body weight (kg) & $65.7 \pm 11.4$ & $66.0 \pm 12.4$ & 0.916 \\
BMI (kg/m²) & $23.8 \pm 2.8$ & $25.1 \pm 2.9$ & 0.058 \\
SBP (mmHg) & $120.2 \pm 16.0$ & $121.7 \pm 18.2$ & 0.696 \\
DBP (mmHg) & $73.8 \pm 12.3$ & $75.3 \pm 11.8$ & 0.614 \\
Glucose (mg/dl) & $90.8 \pm 12.9$ & $147.3 \pm 40.3$ & $<0.001$ \\
HbA1c (\%) & $5.7 \pm 0.3$ & $8.5 \pm 1.8$ & $<0.001$ \\
TC (mg/dl) & $202.6 \pm 34.5$ & $200.1 \pm 39.2$ & 0.764 \\
LDL-C (mg/dl) & $111.2 \pm 24.3$ & $118.3 \pm 31.8$ & 0.243 \\
HDL-C (mg/dl) & $52.6 \pm 12.0$ & $49.6 \pm 10.3$ & 0.272 \\
TG (mg/dl) & $129.0 \pm 67.9$ & $143.3 \pm 65.5$ & 0.255 \\
hs-CRP (mg/dl) & $0.17 \pm 0.52$ & $0.56 \pm 0.98$ & $<0.001$ \\
Sortilin (ng/ml) & $1.34 \pm 1.0$ & $1.57 \pm 0.54$ & 0.006 \\
HTN (\%) & 61.1 & 41.2 & 0.008 \\
Dyslipidemia (\%) & 71.0 & 73.9 & 0.999
\end{tabular}

Data are expressed as mean $\pm S D$, or \%. CAD coronary artery disease, $D M$ diabetes mellitus, $B M I$ body mass index, $S B P$ systolic blood pressure, $D B P$ diastolic blood pressure, TC total cholesterol, LDL-C LDL cholesterol, HDL-C HDL cholesterol, TG triglyceride, HTN hypertension

$P$ values for student's $t$ test or nonparametric $t$ test or Chi square test and total cholesterol to avoid collinearity. Multivariable logistic regression analysis indicated that age, HDL-cholesterol, log hs-CRP, and log sortilin were independently associated with the presence of CAD.

The ability of the area under the receiver operating characteristic (ROC) curve based on sortilin levels to predict the presence of CAD was 0.725 (Fig. 2). The optimal cut-off values of sortilin were $1.35 \mathrm{ng} / \mathrm{ml}(80.6 \%$ sensitivity and $53.0 \%$ specificity) to detect CAD. We performed same analysis in the subgroup without CAD. In this analysis we found that the area under ROC curve for sortilin levels to predict the presence of diabetes mellitus was 0.681 , and the asymptotic significance was 0.007 .

\section{Discussion}

This is the first study to demonstrate that circulating sortilin levels measured by commercial ELISA are associated with both CAD and diabetes mellitus. Subjects with CAD or diabetes mellitus had higher sortilin levels than control subjects, and plasma sortilin levels were independently associated with CAD. In subgroup of subjects free from CAD, circulating sortilin levels were also associated with the presence of diabetes mellitus. These results demonstrated that sortilin levels can be used as a biomarker for $\mathrm{CAD}$ and diabetes mellitus, further studies are required

Table 3 General characteristics of patients by tertiles of circulating sortilin level

\begin{tabular}{|c|c|c|c|c|}
\hline & \multicolumn{3}{|c|}{ Tertiles of circulating sortilin levels } & \multirow[t]{2}{*}{$P$ value } \\
\hline & Tertile $1(<1.17 \mathrm{ng} / \mathrm{ml}) \mathrm{n}=49$ & Tertile $2(1.17-1.59 \mathrm{ng} / \mathrm{ml}) \mathrm{n}=49$ & Tertile $3(\geq 1.60 \mathrm{ng} / \mathrm{ml}) \mathrm{n}=49$ & \\
\hline Age (years) & $50.4 \pm 6.5^{\mathrm{a}}$ & $60.0 \pm 9.9^{b}$ & $56.2 \pm 13.4^{b}$ & $<0.001$ \\
\hline Men (\%) & 73.5 & 67.3 & 67.3 & 0.750 \\
\hline Body weight (kg) & $67.4 \pm 10.2$ & $64.2 \pm 11.1$ & $64.2 \pm 11.5$ & 0.249 \\
\hline $\mathrm{BMI}\left(\mathrm{kg} / \mathrm{m}^{2}\right)$ & $24.1 \pm 2.3$ & $23.9 \pm 3.1$ & $23.8 \pm 3.0$ & 0.890 \\
\hline SBP $(\mathrm{mmHg})$ & $119.9 \pm 16.3$ & $122.8 \pm 15.9$ & $124.0 \pm 18.8$ & 0.473 \\
\hline $\mathrm{DBP}(\mathrm{mmHg})$ & $73.8 \pm 11.7$ & $73.9 \pm 10.5$ & $73.5 \pm 12.9$ & 0.985 \\
\hline Glucose (mg/dl) & $94.0 \pm 1.3^{\mathrm{a}}$ & $113.3 \pm 1.4^{b}$ & $115.1 \pm 1.5^{b}$ & 0.003 \\
\hline $\mathrm{HbA1c}(\%)$ & $5.9 \pm 1.1^{\mathrm{a}}$ & $6.3 \pm 1.2^{\mathrm{a}}$ & $6.6 \pm 1.3^{b}$ & 0.011 \\
\hline $\mathrm{TC}(\mathrm{mg} / \mathrm{dl})$ & $202.2 \pm 34.9$ & $191.7 \pm 41.5$ & $188.2 \pm 43.5$ & 0.206 \\
\hline LDL-C (mg/dl) & $111.1 \pm 22.5$ & $109.9 \pm 25.3$ & $109.3 \pm 32.3$ & 0.946 \\
\hline $\mathrm{HDL}-\mathrm{C}(\mathrm{mg} / \mathrm{dl})$ & $51.5 \pm 13.7$ & $49.7 \pm 10.9$ & $46.1 \pm 11.9$ & 0.082 \\
\hline $\mathrm{TG}(\mathrm{mg} / \mathrm{dl})$ & $122.0 \pm 1.6$ & $110.8 \pm 1.7$ & $118.9 \pm 1.7$ & 0.631 \\
\hline hs-CRP (mg/dl) & $0.10 \pm 0.15$ & $0.28 \pm 0.56$ & $0.49 \pm 1.09$ & 0.015 \\
\hline $\mathrm{DM}(\%)$ & 8.2 & 34.7 & 44.9 & $<0.001$ \\
\hline CAD (\%) & 0 & 26.5 & 36.7 & $<0.001$ \\
\hline HTN (\%) & 14.3 & 75.5 & 71.4 & $<0.001$ \\
\hline Dyslipidemia (\%) & 30.6 & 32.7 & 49.0 & 0.120 \\
\hline
\end{tabular}

Data are expressed as mean $\pm S D$, or \%. BMI body mass index, SBP systolic blood pressure, $D B P$ diastolic blood pressure, $T C$ total cholesterol, $L D L-C L D L$ cholesterol, $H D L-C H D L$ cholesterol, $T G$ triglyceride, $D M$ diabetes mellitus, CAD coronary artery disease, $H T N$ hypertension

Log-transformation was used for variables (glucose, $\mathrm{HbA} 1 \mathrm{c}$, triglyceride, hs-CRP, and sortilin) before statistical analysis. $P$ values for one-way analysis of variance (ANOVA)

a,b The data with different superscript letters are significantly different 
Table 4 Univariable and multivariable logistic regression analyses for coronary artery disease

\begin{tabular}{|c|c|c|c|c|}
\hline & \multicolumn{4}{|c|}{ Association with presence of CAD } \\
\hline & \multicolumn{2}{|l|}{ Single } & \multicolumn{2}{|l|}{ Multiple } \\
\hline & OR $(95 \% \mathrm{Cl})$ & $P$ value & OR $(95 \% \mathrm{Cl})$ & $P$ value \\
\hline Age (years) & $1.14(1.08-1.20)$ & $<0.001$ & $1.08(1.00-1.17)$ & 0.04 \\
\hline $\mathrm{BMI}\left(\mathrm{kg} / \mathrm{m}^{2}\right)$ & $0.91(0.79-1.06)$ & 0.213 & - & - \\
\hline SBP $(\mathrm{mmHg})$ & $1.03(1.00-1.05)$ & 0.021 & $1.03(0.98-1.08)$ & 0.262 \\
\hline $\mathrm{DBP}(\mathrm{mmHg})$ & $0.99(0.85-1.02)$ & 0.435 & - & - \\
\hline Log glucose (mg/dl) & $41.69(9.19-189.12)$ & $<0.001$ & - & - \\
\hline Log HbA1c (\%) & $10.06(1.54-65.87)$ & 0.016 & $0.12(0.02-7.61)$ & 0.124 \\
\hline $\mathrm{TC}(\mathrm{mg} / \mathrm{dl})$ & $0.97(0.96-0.99)$ & $<0.001$ & - & - \\
\hline $\log$ TG (mg/dl) & $1.13(0.52-2.48)$ & 0.757 & - & - \\
\hline $\mathrm{HDL}-\mathrm{C}(\mathrm{mg} / \mathrm{dl})$ & $0.86(0.81-0.91)$ & $<0.001$ & $0.82(0.72-0.93)$ & 0.002 \\
\hline LDL-C (mg/dl) & $0.98(0.97-1.00)$ & 0.028 & $1.01(0.98-1.05)$ & 0.513 \\
\hline Log hs-CRP (mg/dl) & $2.64(1.80-3.88)$ & $<0.001$ & $2.27(1.28-4.04)$ & 0.005 \\
\hline Log sortilin (ng/ml) & $4.17(1.68-10.35)$ & 0.002 & $5.29(1.06-26.51)$ & 0.043 \\
\hline
\end{tabular}

$C A D$ coronary artery disease, $D M$ diabetes mellitus, $B M I$ body mass index, $S B P$ systolic blood pressure, $D B P$ diastolic blood pressure, $T C$ total cholesterol, $T G$ triglyceride, HDL-C HDL cholesterol, LDL-C LDL cholesterol

Log-transformation was used for variables (glucose, $\mathrm{HbA1c}$, triglyceride, hs-CRP, and sortilin) before statistical analysis. $P$ values for univariable or multivariable logistic regression analysis

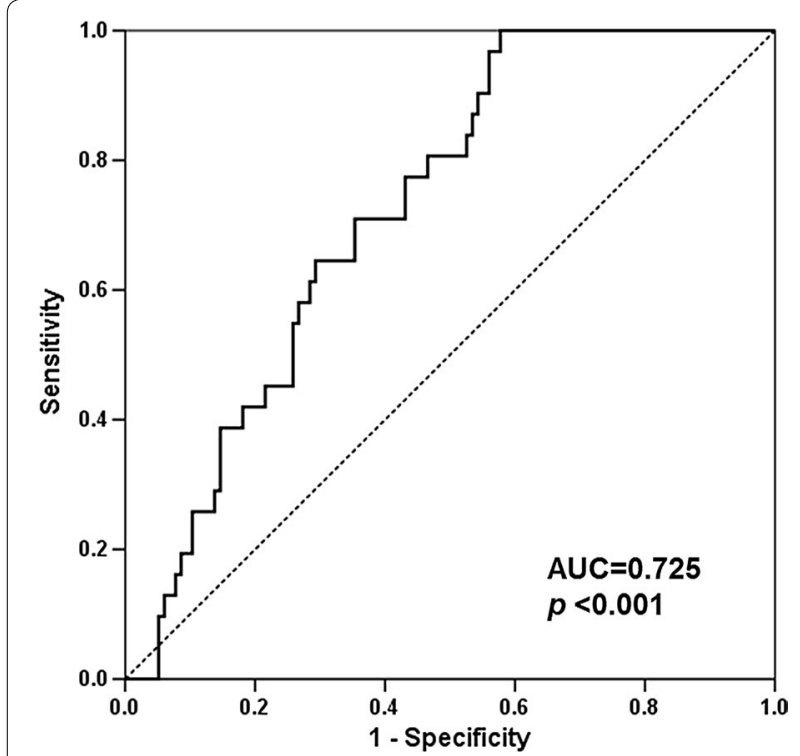

Fig. 2 ROC curve analysis of the ability of sortilin to predict the presence of coronary artery disease

to validate these results and to determine the relationship between sortilin and glucose metabolism.

Sortilin is one of three neurotensin receptors, neurotensin receptor-3 [16]. The level of proneurotensin, the propeptide of neurotensin, was reported to be related to the risk of incident diabetes mellitus in a prospective cohort study [17]. In this cohort, individuals with higher circulating proneurotensin had a higher incidence of diabetes mellitus, with a hazard ratio of 1.28 (95\% confidence interval 1.07-1.50, $P=0.003$ ). In addition, in another human study, plasma proneurotensin levels were higher in insulin-resistant obese subjects [18]. In our study, plasma levels of sortilin, a receptor for neurotensin, were related to diabetes mellitus and insulin resistance. Therefore, sortilin/neurotensin signaling might play an important role in insulin signaling. One possible explanation for this is suggested by the role of sortilin in glucose transporter-4 translocation in adipocytes [19]. Furthermore, neurotensin deficient mice were resistant to high-fat diet-induced obesity, and neurotensin decreases AMP-activated protein kinase activity via sortilin [18]. Our study results add new evidence for the link between sortilin and insulin signaling and glucose regulation.

Our results are noteworthy because we included subjects using strict criteria that minimized possible bias. First, we excluded subjects taking statin therapy. A previous study showed that treatment with pitavastatin or pravastatin for 8 months significantly decreased the circulating sortilin levels compared with baseline by $8 \%$ for pitavastatin and $16 \%$ for pravastatin [20]. Therefore, statin therapy has an impact on circulating sortilin levels, and we eliminated this bias by including only statin-naïve subjects. Second, we defined a normal coronary artery based on coronary CT angiography, a test that has a high negative predictive value [21]. Therefore, our data were not confounded by uncertainty about the presence of 
CAD, in contrast to other studies that define the absence of CAD from the subjects' medical history. Third, we analyzed the data by several statistical methods, and the results were consistent.

Genome-wide association studies have provided good insight into the relationship between lipid metabolism and $\mathrm{CAD}[22,23]$. From these studies the cardiovascular phenotype of genetic variants seems to depend on ethnicity or the presence of diabetes mellitus. Variants in the SORT1 locus also showed an association with lipid metabolism and CAD [24]. However, the effect of this genetic variant on blood sortilin levels has not been clarified. In contrast to our data, a Japanese study [14] showed that the soluble sortilin levels were lower in subjects with CAD than in controls. The Japanese study used a different type of assay to measure the sortilin levels, and the majority of study subjects were being treated with statins. Therefore, when we used sortilin as a biomarker, we made an effort to standardize the measurement of blood sortilin levels and elucidate the impact of various medications on its levels.

Sortilin is expressed widely, including in the nervous system, liver, adipose tissue, and in immune cells including macrophages [25]. Whole-body sortilin-knockout mice had decreased plasma HDL- and LDL-cholesterol levels accompanied by attenuation of atherosclerotic lesions [10] and vascular calcification [26]. Similarly, macrophage sortilin-deficient mice showed reduced atherosclerotic lesions because of reduced LDL uptake by macrophages [27]. In contrast, serum LDL-cholesterol levels were decreased rather than increased after overexpression of sortilin in the liver using adenovirus [28]. This study used sortilin molecules including the C-terminus, and this form of sortilin is likely to include the transmembrane region. This type of sortilin enhanced LDL clearance via an LDL receptor-independent pathway. A subsequent study demonstrated that hepatic sortilin reduces hepatic apolipoprotein $\mathrm{B}$ secretion and increases LDL catabolism [29]. In contrast, another study using sortilin without the $\mathrm{C}$-terminal region enhanced hepatic very low density lipoprotein (VLDL), leading to increased LDL levels in the blood [10]. Therefore, the role of sortilin in lipid metabolism seems to be differ according to its structure, location, and target tissue. To our knowledge, there are no previous studies of the correlation between tissue and circulating levels of sortilin. In our study, we confirmed that higher circulating sortilin levels were related to an increased risk of CAD. However, we did not see any significant correlation between circulating sortilin levels and LDL-cholesterol or hepatic sortilin content. In the case of LDL-cholesterol, one type of sortilin can reduce VLDL secretion, while the other type of sortilin can enhance internalization of LDL-cholesterol.
In addition, the exact role of circulating sortilin, the type measured in our study, is not known. There may be mechanisms other than lipid metabolism that contribute to the association between circulating sortilin and CAD. Interestingly, in the subgroup of subjects without CAD the insulin resistance calculated by HOMA-IR was positively correlated with circulating sortilin levels (Spearman's $\rho=0.513, P=0.015$ ). Insulin resistance could be a link between sortilin, atherosclerosis and diabetes mellitus. Further mechanistic studies are needed to confirm this hypothesis.

\section{Limitations}

Our study has several limitations. First, this is a crosssectional study, so we could not predict future development of CAD and diabetes mellitus. The suggested cut-off value of sortilin for discriminating CAD had relatively low specificity, so we were unable to diagnose CAD solely based on blood sortilin level. A large-scale prospective cohort study is needed to confirm the usefulness of circulating sortilin for screening of CAD. In addition, usefulness of sortilin to screen or predict diabetes mellitus is doubtful, because measuring plasma glucose level is more practical than assaying for sortilin. Second, we did not analyze the sortilin levels according to disease severity, e.g., comparing subclinical and clinical atherosclerosis, or prediabetes to overt diabetes. Therefore, we cannot generalize our data to a broad range of CAD including subclinical atherosclerosis. Third, even though we adjusted for the age difference between groups, the age discrepancy could be a confounder. Furthermore, statistical analysis for DM was not feasible because of the small number of subjects without diabetes mellitus who underwent $C A B G$. Fourth, we did not investigate the influence of antidiabetic and antihypertensive medication. Finally, we had no data concerning the presence of single nucleotide polymorphisms of sortilin.

\section{Conclusions}

Circulating sortilin level was associated with both CAD and diabetes mellitus. Elevated circulating sortilin levels were an independent risk factor for CAD and could be a useful biomarker for these two diseases, especially in statin-naïve subjects.

\footnotetext{
Abbreviations

ANOVA: analysis of variance; CABG: coronary artery bypass graft; CAD: coronary artery disease; LSD: least significant difference.

Authors' contributions

OTJ analyzed the data, wrote the manuscript. ACH and LC contributed to data collection. KBR performed laboratory experiment. KKM, MJH, and LS contributed in discussion. PKS and JHC participated in study design. CSH designed the study and wrote the manuscript. All authors read and approved the final manuscript.
} 


\begin{abstract}
Author details
1 Department of Internal Medicine, Seoul National University College of Medicine, Seoul, South Korea. ${ }^{2}$ Department of Internal Medicine, Seoul National University Bundang Hospital, 300, Gumi-dong, Bundang-gu, Seongnam 463-070, South Korea. ${ }^{3}$ Department of Thoracic and Cardiovascular Surgery, Seoul National University College of Medicine, Seoul, South Korea. ${ }^{4}$ Department of Thoracic and Cardiovascular Surgery, Seoul National University Bundang Hospital, Seongnam, South Korea.
\end{abstract}

\section{Acknowledgements}

The authors thank Division of Statistics in Medical Research Collaborating Center at Seoul National University Bundang Hospital for statistical analyses.

\section{Competing interests}

The authors declare that they have no competing interests.

\section{Availability of data and materials}

All data generated or analyzed during this study are included in this published article.

\section{Consent for publication}

If the manuscript is accepted, we approve it for publication in Cardiovascular Diabetology

\section{Ethics approval and consent to participate}

All participants provided written informed consent and the study was performed under the approval of the Institutional Review Board of the SNUBH.

\section{Funding}

Financial support was provided through the Korean Health Technology R\&D Project, Ministry of Health \& Welfare, Republic Korea (HI14C0074).

\section{Publisher's Note}

Springer Nature remains neutral with regard to jurisdictional claims in published maps and institutional affiliations.

Received: 17 March 2017 Accepted: 22 June 2017

Published online: 20 July 2017

\section{References}

1. Sajankila N, Como JJ, Claridge JA. Upcoming rules and benchmarks concerning the monitoring of and the payment for surgical infections. Surg Clin North Am. 2014;94(6):1219-31.

2. Shaw JE, Sicree RA, Zimmet PZ. Global estimates of the prevalence of diabetes for 2010 and 2030. Diabetes Res Clin Pract. 2010;87(1):4-14.

3. Ortega Moreno L, Copetti M, Fontana A, De Bonis C, Salvemini L, Trischitta $\checkmark$, et al. Evidence of a causal relationship between high serum adiponectin levels and increased cardiovascular mortality rate in patients with type 2 diabetes. Cardiovasc Diabetol. 2016;15:17.

4. Gregg EW, Li Y, Wang J, Burrows NR, Ali MK, Rolka D, et al. Changes in diabetes-related complications in the United States, 1990-2010. N Engl J Med. 2014;370(16):1514-23.

5. Grundy SM, Benjamin IJ, Burke GL, Chait A, Eckel RH, Howard BV, et al. Diabetes and cardiovascular disease: a statement for healthcare professionals from the American Heart Association. Circulation. 1999;100(10):1134-46.

6. Adela R. GDF-15 as a target and biomarker for diabetes and cardiovascular diseases: a translational prospective. J Diabetes Res. 2015. doi:10.1155/2015/490842.

7. Odegaard AO, Jacobs DR Jr, Sanchez OA, Goff DC Jr, Reiner AP, Gross MD. Oxidative stress, inflammation, endothelial dysfunction and incidence of type 2 diabetes. Cardiovasc Diabetol. 2016;15:51.

8. von Scholten BJ, Reinhard H, Hansen TW, Lindhardt M, Petersen CL, Wiinberg $\mathrm{N}$, et al. Additive prognostic value of plasma N-terminal pro-brain natriuretic peptide and coronary artery calcification for cardiovascular events and mortality in asymptomatic patients with type 2 diabetes. Cardiovasc Diabetol. 2015;14:59.
9. Samani NJ, Erdmann J, Hall AS, Hengstenberg C, Mangino M, Mayer B, et al. Genomewide association analysis of coronary artery disease. N Engl J Med. 2007;357(5):443-53.

10. Kjolby M, Andersen OM, Breiderhoff T, Fjorback AW, Pedersen KM, Madsen P, et al. Sort1, encoded by the cardiovascular risk locus 1 1 13.3, is a regulator of hepatic lipoprotein export. Cell Metab. 2010;12(3):213-23.

11. Kathiresan S, Melander O, Guiducci C, Surti A, Burtt NP, Rieder MJ, et al. Six new loci associated with blood low-density lipoprotein cholesterol, highdensity lipoprotein cholesterol or triglycerides in humans. Nat Genet. 2008;40(2):189-97.

12. Hermey G. The Vps10p-domain receptor family. Cell Mol Life Sci. 2009;66(16):2677-89.

13. Navarro V, Vincent JP, Mazella J. Shedding of the luminal domain of the neurotensin receptor-3/sortilin in the HT29 cell line. Biochem Biophys Res Commun. 2002;298(5):760-4.

14. Ogawa K, Ueno T, Iwasaki T, Kujiraoka T, Ishihara M, Kunimoto S, et al. Soluble sortilin is released by activated platelets and its circulating levels are associated with cardiovascular risk factors. Atherosclerosis. 2016;249:110-5.

15. Januzzi JL Jr, Lyass A, Liu Y, Gaggin H, Trebnick A, Maisel AS, et al. Circulating proneurotensin concentrations and cardiovascular disease events in the community: the Framingham Heart Study. Arterioscler Thromb Vasc Biol. 2016;36(8):1692-7.

16. Vincent JP, Mazella J, Kitabgi P. Neurotensin and neurotensin receptors. Trends Pharmacol Sci. 1999;20(7):302-9.

17. Melander O, Maisel AS, Almgren P, Manjer J, Belting M, Hedblad B, et al. Plasma proneurotensin and incidence of diabetes, cardiovascular disease, breast cancer, and mortality. JAMA. 2012;308(14):1469-75.

18. Li J, Song J, Zaytseva YY, Liu Y, Rychahou P, Jiang K, et al. An obligatory role for neurotensin in high-fat-diet-induced obesity. Nature. 2016;533(7603):411-5.

19. Morris NJ, Ross SA, Lane WS, Moestrup SK, Petersen CM, Keller SR, et al. Sortilin is the major 110-kDa protein in GLUT4 vesicles from adipocytes. J Biochem. 1998;273(6):3582-7.

20. Nozue T, Hattori H, Ogawa K, Kujiraoka T, Iwasaki T, Michishita I. Effects of statin therapy on plasma proprotein convertase subtilisin/kexin type 9 and sortilin levels in statin-naive patients with coronary artery disease. J Atheroscler Thromb. 2016;23(7):848-56.

21. Leber AW, Knez A, Becker A, Becker C, von Ziegler F, Nikolaou K, et al. Accuracy of multidetector spiral computed tomography in identifying and differentiating the composition of coronary atherosclerotic plaques: a comparative study with intracoronary ultrasound. J Am Coll Cardiol. 2004;43(7):1241-7.

22. Kim YK, Hwang MY, Kim YJ, Moon S, Han S, Kim BJ. Evaluation of pleiotropic effects among common genetic loci identified for cardio-metabolic traits in a Korean population. Cardiovasc Diabetol. 2016:15:20.

23. Beaney KE, Cooper JA, McLachlan S, Wannamethee SG, Jefferis BJ, Whincup P, et al. Variant rs 10911021 that associates with coronary heart disease in type 2 diabetes, is associated with lower concentrations of circulating HDL cholesterol and large HDL particles but not with amino acids. Cardiovasc Diabetol. 2016;15(1):115.

24. Strong A, Patel K, Rader DJ. Sortilin and lipoprotein metabolism: making sense out of complexity. Curr Opin Lipidol. 2014;25(5):350-7.

25. Zhong LY, Cayabyab FS, Tang CK, Zheng XL, Peng TH, LV YC. Sortilin: a novel regulator in lipid metabolism and atherogenesis. Clinica Chimica Acta. 2016:460:11-7.

26. Goettsch C, Hutcheson JD, Aikawa M, Iwata H, Pham T, Nykjaer A, et al. Sortilin mediates vascular calcification via its recruitment into extracellular vesicles. J Clin Investig. 2016;126(4):1323-36.

27. Patel KM, Strong A, Tohyama J, Jin X, Morales CR, Billheimer J, et al. Macrophage sortilin promotes LDL uptake, foam cell formation, and atherosclerosis. Circ Res. 2015;116(5):789-96.

28. Musunuru K, Strong A, Frank-Kamenetsky M, Lee NE, Ahfeldt T, Sachs $\mathrm{KV}$, et al. From noncoding variant to phenotype via SORT1 at the $1 \mathrm{p} 13$ cholesterol locus. Nature. 2010;466(7307):714-9.

29. Strong A, Ding Q, Edmondson AC, Millar JS, Sachs KV, Li X, et al. Hepatic sortilin regulates both apolipoprotein B secretion and LDL catabolism. J Clin Investig. 2012;122(8):2807-16. 